\title{
Nanomedicine developments in the treatment of metastatic pancreatic cancer: focus on nanoliposomal irinotecan
}

This article was published in the following Dove Press journal:

International Journal of Nanomedicine

31 March 2016

Number of times this article has been viewed

\author{
Andrew $\mathrm{H} \mathrm{Ko}$ \\ Division of Hematology/Oncology, \\ Helen Diller Family Comprehensive \\ Cancer Center, University of \\ California, San Francisco, San \\ Francisco, CA, USA
}

\begin{abstract}
Nanoliposomal irinotecan (nal-IRI) was originally developed using an efficient and high-loading capacity system to encapsulate irinotecan within a liposomal carrier, producing a therapeutic agent with improved biodistribution and pharmacokinetic characteristics compared to free drug. Specifically, administration of nal-IRI results in prolonged exposure of SN-38, the active metabolite of irinotecan, within tumors, while at the same time offering the advantage of less systemic toxicity than traditional irinotecan. These favorable properties of nal-IRI, confirmed in a variety of tumor xenograft models, led to its clinical evaluation in a number of disease indications for which camptothecins have proven activity, including in colorectal, gastric, and pancreatic cancers. The culmination of these clinical trials was the NAPOLI-1 (Nanoliposomal irinotecan with fluorouracil and folinic acid in metastatic pancreatic cancer after previous gemcitabine-based therapy) trial, an international Phase III study evaluating nal-IRI both alone and in combination with 5-fluorouracil and leucovorin in patients with metastatic pancreatic adenocarcinoma following progression on gemcitabine-based chemotherapy. Positive results from NAPOLI-1 led to approval of nal-IRI (with 5-fluorouracil/leucovorin) in October 2015 by the US Food and Drug Administration specifically for the treatment of metastatic pancreatic cancer in the second-line setting and beyond, a clinical context in which there had previously been no accepted standard of care. As such, nal-IRI represents an important landmark in cancer drug development, and potentially ushers in a new era where a greater number of patients with advanced pancreatic cancer can be sequenced through multiple lines of therapy translating into meaningful improvements in survival.
\end{abstract}

Keywords: pancreatic cancer, irinotecan, nanoliposomal, clinical trial, NAPOLI-1

\section{Introduction}

Pancreatic cancer currently represents the fourth leading cause of cancer-related mortality in the United States among both males and females, with an estimated 48,960 new cases and 40,560 deaths attributed to this malignancy in $2015 .{ }^{1}$ Moreover, the number of pancreatic cancer-related deaths continues to increase, with current projections indicating it will likely rise to the second-leading cause of cancer-related mortality in the United States sometime during the next decade, trailing only lung cancer. ${ }^{2}$ Globally, pancreatic cancer ranks as the seventh most common cause of cancer death, responsible for more than 330,000 deaths worldwide as of 2012 (4\% of the total). ${ }^{3}$

These statistical findings reflect the sobering realities of this disease: the vast majority of patients present at advanced stages of disease beyond which a potentially curative operation is feasible; and therapeutic gains in this disease have been more modest rather than transformative over the past several decades. Moreover, while our understanding of the fundamental pathogenic and molecular bases of pancreatic 
cancer continues to grow, an ability to translate these findings into "actionable" results has been limited, ${ }^{4}$ and we still lack useful predictive biomarkers that can aid in decision-making and bring our treatment paradigms into the modern realm of precision medicine.

On a more positive note, over the past several years we have seen positive results from multiple Phase III clinical trials that have expanded our therapeutic options for patients with advanced pancreatic cancer. The most recent of these developments has led to the approval by the US Food and Drug Administration (FDA) of nanoliposomal irinotecan (herein referred to as nal-IRI) in October 2015. This represents a significant milestone as this drug represents the first agent specifically approved for use in the second-line treatment of metastatic pancreatic cancer.

This paper will start by reviewing briefly the current treatment landscape for pancreatic cancer, with special attention on the proven therapeutic options (or, more accurately, lack thereof) that exist beyond frontline chemotherapy, as this is the specific indication for which nanoliposomal irinotecan has been approved. This will be followed by a discussion of the preclinical development of nal-IRI and its pharmacologic properties; and finally clinical evaluation of nal-IRI from early dose-finding studies to disease-specific clinical trials, with a particular focus on the development and approval of this novel agent in pancreatic cancer.

\section{The current treatment landscape for metastatic pancreatic cancer}

Chemotherapy remains the mainstay of treatment for patients diagnosed with metastatic pancreatic cancer. Single-agent gemcitabine became the standard of care back in the mid1990s following results of a Phase III study that demonstrated improvements in overall survival (OS), response rate, as well as a quality of life measure termed clinical benefit response (a composite measure of analgesic requirements, performance status, and change in weight) when compared with 5-fluorouracil (5-FU). ${ }^{5}$ Over the subsequent decade plus, a number of Phase III trials were designed to assess whether any survival benefit could be derived from adding additional cytotoxic or targeted agents to gemcitabine. With the exception of the PA.3 trial, which evaluated erlotinib, an oral tyrosine kinase inhibitor that binds to the epidermal growth factor receptor (EGFR), ${ }^{6}$ none of these studies demonstrated a statistically significant survival advantage of combination therapy when compared to gemcitabine alone. Moreover, despite approval of erlotinib by the FDA in 2005, its use has not been very widespread, based on a fairly modest incremental survival benefit when added to gemcitabine (hazard ratio (HR) of 0.81 for overall survival) and substantial toxicities.

After this relatively fallow period through the early 2000s, positive data from a couple of randomized Phase III trials over the past several years have led to the acceptance of two new standards of care for the first-line treatment of metastatic pancreatic cancer. PRODIGE 4/ACCORD 11 was a 342-patient French trial in which FOLFIRINOX, a multidrug combination consisting of bolus plus infusional 5-FU, leucovorin (LV), irinotecan, and oxaliplatin administered on a biweekly dosing schedule, was compared to single-agent gemcitabine in patients with previously untreated metastatic pancreatic cancer and intact functional status (Eastern Cooperative Oncology Group performance status of $0-1){ }^{7}$ This study unequivocally demonstrated the superiority of FOLFIRINOX in terms of all clinically relevant parameters, including prolongation of overall survival (median OS: 11.1 vs 6.8 months, HR of $0.57 ; P<0.001$ ) and progressionfree survival, higher objective response rate, and longer preservation of quality of life. ${ }^{8}$ As a result, FOLFIRINOX has become the preferred first-line choice for select patients who are robust enough to tolerate this somewhat more aggressive chemotherapy regimen.

A second chemotherapy platform now in widespread use for metastatic pancreatic cancer consists of the combination of gemcitabine plus nab-paclitaxel (Celgene, Summit, NJ, USA), a 130-nm albumin-bound formulation of paclitaxel particles. Nab-paclitaxel, originally FDA-approved for advanced breast cancer back in 2005, was developed in part to eliminate the allergic reactions that could occur from polyoxyethylated castor oil (Cremphor EL; BASF, Ludwigshafen, Germany), a vehicle needed for parenteral administration of free paclitaxel. Its subsequent evaluation in pancreatic cancer was based in part on molecular profiling of pancreatic tumor samples, in which overexpression of the SPARC protein (secreted protein acidic and rich in cysteine), an albumin-binding protein, was observed. ${ }^{9}$ Following promising results from a Phase I/II trial of gemcitabine plus nab-paclitaxel, ${ }^{10}$ an international Phase III study (the MPACT trial) was conducted in which 861 patients with metastatic pancreatic cancer were randomized to receive gemcitabine either alone or in combination with nab-paclitaxel. ${ }^{11}$ Patients receiving the doublet had superior outcomes, including a significant prolongation of survival (median OS: 8.5 vs 6.7 months, $\mathrm{HR}=0.72 ; P<0.001$ ), thus leading to FDA approval of nab-paclitaxel for this indication in 2014. 


\section{What options do we currently have beyond frontline chemotherapy?}

Data from contemporary pancreatic cancer trials suggest that fewer than half of patients receiving first-line chemotherapy go on to receive any additional therapy; for example, in the aforementioned MPACT trial, only $40 \%$ of patients received second-line treatment. ${ }^{11}$ This reflects both the often rapid clinical deterioration of patients at this stage of their disease trajectory as well as the lack of available therapeutic options in the salvage setting. The emergence of two frontline standards for metastatic pancreatic cancer may now afford greater opportunity to sequence patients across multiple lines of therapy. It is important, however, to recognize that randomized clinical trial data supporting a sequential approach of FOLFIRINOX followed by gemcitabine/nab-paclitaxel (or the reverse) are still lacking, with only small series (primarily retrospective) and case reports suggesting modest efficacy of each of these regimens in the second-line setting. ${ }^{12-16}$

A variety of other therapies, including not only classical cytotoxic drugs (alone and in combination) but also molecularly targeted agents and, more recently, immunotherapies, have been investigated in this salvage setting. One systematic review published in 2013 evaluating 34 clinical trials of different second-line regimens following gemcitabine-based therapy concluded that continuing with some form of therapy after progression on first-line treatment did confer a survival advantage when compared to best supportive care, ${ }^{17}$ thus supporting this strategy as a general principle. However, the challenges of interpreting this mix of studies include small sample sizes, nonrandomized trial design for the majority with resultant selection bias, and sometimes conflicting results. For example, the de facto standard of care for many years when treating patients with gemcitabine-resistant pancreatic cancer was to move next to a combination of a platinum analog plus a fluoropyrimidine. This approach was informed in large part by results from one of the largest randomized studies (a German trial called CONKO-003) in this disease setting demonstrating a survival advantage of OFF (oxaliplatin, folinic acid, and fluorouracil) compared to FF alone (median OS: 5.9 vs 3.3 months, $\mathrm{HR}=0.66$; log-rank $P=0.010) .{ }^{18}$ Conversely, a later trial conducted in Canada called PANCREOX showed almost the precise opposite results in a similar patient population, with patients treated with FOLFOX (chemotherapy regimen of folinic acid, 5-FU, and oxaliplatin) faring no better - and possibly worse - than those receiving 5-FU/LV alone. ${ }^{19}$

In addition to nal-IRI, several other novel agents have shown promising results in the second-line (and beyond) setting for metastatic pancreatic cancer and are moving ahead in clinical development. In the randomized Phase II RECAP trial, ${ }^{20}$ the JAK 1/2 inhibitor ruxolitinib (Jakafi, Incyte Pharmaceuticals, New York, NY, USA) was evaluated in combination with capecitabine in patients who had failed gemcitabine-based chemotherapy. In a preplanned analysis of the subgroup of patients with elevated levels of C-reactive protein (CRP) (an indicator of high levels of systemic inflammation that contributes to cancer-related cachexia), median survival was significantly longer in those patients who received the ruxolitinib/capecitabine combination compared to capecitabine alone ( 83 vs 55 days, $\mathrm{HR}=0.47 ; P=0.01$ ). These results have led to two Phase III trials (JANUS 1 and JANUS 2) that are currently underway to evaluate ruxolitinib specifically in gemcitabine-refractory pancreatic cancer patients with high CRP levels. A second, immune-based approach being tested in this setting is CRS-207 (Aduro Biosciences, Berkeley, CA, USA), a live-attenuated Listeria monocytogenes vaccine vector genetically engineered to express the tumor-associated antigen, mesothelin. Immunotherapeutic strategies have not produced as dramatic results in pancreatic cancer when compared with other solid malignancies, perhaps due to pancreatic cancer being generally considered a more immune-privileged tumor. ${ }^{21}$ Nevertheless, in a randomized Phase II trial in patients with chemorefractory metastatic pancreatic cancer, CRS-207, when combined with the cellular vaccine GVAX, significantly improved survival when compared to GVAX alone (median OS: 6.0 vs 3.4 months, $\mathrm{HR}=0.4477 ; P=0.0057$ ), including in some patients with very prolonged disease stabilizations. ${ }^{22}$ These results have led to successor trials comparing this vaccinebased strategy to chemotherapy, as well as evaluating it in combination with immune checkpoint blockade, in the second- and third-line settings.

\section{Introduction to nal-IRI: initial drug development}

Liposomes are spherical drug carrier vehicles with a bilayer lipid membrane, typically ranging in size anywhere from approximately $40 \mathrm{~nm}$ to several microns. ${ }^{23}$ The theoretical benefits of developing liposomal formulations of anticancer drugs, including both traditional chemotherapy agents as well as molecularly targeted therapies (such as small molecule inhibitors), are well-established; these include the possibility of encapsulating poorly soluble drugs, protecting therapeutic agents from premature clearance and metabolism, and improving biodistribution and pharmacokinetics via slow release of the parent agent (reviewed in Bertrand 
et $\mathrm{al}^{24}$ ), resulting in simultaneously greater potency and decreased side effects. Ideally, liposomes should retain encapsulated drug in circulation and then release the drug in a time-controlled fashion after arrival at the target tissue. This release rate is an important factor in drug potency that can be controlled, to some extent, by various physiochemical properties of the liposome, including both lipid and interior buffer composition. ${ }^{25}$

It is believed that nanoliposomes accumulate preferentially in the tumor through a phenomenon known as the enhanced permeability and retention (EPR) effect. ${ }^{26}$ This EPR effect describes the combination of irregular, permeable tumor blood vessels with large fenestrations that enable extravasation of macromolecules (such as liposomes), together with impaired lymphatic drainage. These findings in concert lead to increased cumulative trapping of liposomal drug in the tumor microenvironment, relative to other tissues, in what could be characterized as a form of passive targeting. Once deposited in the tumor, the liposomal agent can then be taken up by tumor-resident macrophages, which release the bound drug resulting in high intratumoral levels of active drug. ${ }^{27}$ The final result is improved pharmacological potency of the liposomal formulation compared to the same drug administered in free form.

At the same time, some technical challenges persist in liposomal technology, including the efficient loading of cancer drugs at high drug-to-lipid ratios, and preventing phagocytosis of liposome-plasma protein complexes by the reticuloendothelial system. Newer stealth technologies, such as the incorporation of polyethylene glycol (PEG) to the liposome surface, are being applied to prevent binding of liposomes by circulating plasma proteins with the goal of decreasing elimination from the circulation.

The therapeutic efficacy of liposomal agents in oncology has been established in routine clinical practice, with agents such as liposome-encapsulated doxorubicin currently being used for a variety of malignant indications (Kaposi sarcoma, multiple myeloma, ovarian cancer), offering the advantage of decreased cardiac toxicity of the cytotoxic drug. However, specific to pancreatic cancer, only a small number of liposomally formulated agents have been tested in the clinical setting, with none gaining significant traction. A PEGylated liposomal formulation of cisplatin (lipoplatin) was evaluated in a small Phase I/II trial in combination with gemcitabine for patients with previously treated advanced pancreatic cancer; efficacy was modest, with two of 24 subjects $(8.3 \%)$ achieving an objective response, and an additional 14 patients $(58.3 \%)$ demonstrating stable disease for a median duration of 3 months. ${ }^{28} \mathrm{~A}$ larger randomized Phase II trial evaluating a novel cationic liposomal formulation of paclitaxel (EndoTAG-1) in combination with gemcitabine as first-line treatment for patients with advanced pancreatic cancer showed somewhat greater promise. ${ }^{29}$ The investigators reported (in abstract form only) a survival benefit with the addition of EndoTAG-1 to gemcitabine, with an HR for overall survival of 0.67 when the highest dose level of this agent was administered together with gemcitabine compared to gemcitabine alone. Nevertheless, neither of these liposomal agents went on to further clinical development in pancreatic cancer.

nal-IRI (originally referred to as PEP-02 when licensing rights were owned by PharmaEngine [Taipei, Taiwan]; subsequently relicensed as MM-398 [Merrimack Pharmaceuticals, Cambridge, MA, USA]), represents a novel nanoliposomal formulation of irinotecan hydrochloride, a semisynthetic analog of the natural alkaloid camptothecin currently used in the treatment of a wide variety of malignancies, including colorectal, gastroesophageal, small cell lung, and breast cancer. Irinotecan, by stabilizing the complex between topoisomerase I (TOP1) and bound DNA, induces stalling of replication forks, ultimately leading to DNA strand breaks and inhibition of replication. Time of drug exposure is an important driver for its cytotoxic effects, ${ }^{30}$ suggesting that irinotecan and other TOP1-targeting agents, in liposomal form, would be able to take particular advantage of the EPR effect with preferential intratumoral accumulation and exposure.

The dose-limiting toxicities (DLTs) of irinotecan are well-described, most notably diarrhea (which can be severe) and cytopenias. nal-IRI was designed in part to reduce the incidence and severity of these toxicities while maintaining or increasing the antitumor activity of the parent drug, offering an improved therapeutic window. Specifically, the liposomal carrier system offers a way to protect irinotecan from premature conversion by nonspecific carboxylesterases into its active metabolite, SN-38, which is about 100- to 1,000-fold more potent. ${ }^{31}$ This produces lower maximum plasma concentration $\left(C_{\max }\right)$ and consequently reduced drug toxicity, even while lower drug elimination prolongs systemic circulation of the liposomal construct. Despite these theoretical advantages, prior liposomal formulations of irinotecan, as well as of other camptothecins (lurtotecan and SN-38, among others), have not necessarily shown improved pharmacokinetic characteristics or toxicity profiles compared to their free drug counterparts when evaluated in the preclinical setting. ${ }^{32-34}$ 
The development of nal-IRI was originally described in 2006 by Drummond et al, ${ }^{35}$ who used a novel intraliposomal drug stabilization technique to load irinotecan into a nanoparticle/liposome construct in efficient manner. Specifically, a polyalkylammonium salt of a nonpolymeric (sucrose octasulfate) highly charged multivalent anion was employed as an intraliposomal trapping agent, resulting in formation of intraliposomal drug-polyanion complexes. (A polymeric agent, polyphosphate, was also tested for synthesis of this drug; but the superior results of sucrose octasulfate led to this being the choice for subsequent clinical development). The triethylammonium component of the salt ensures the charge neutrality of the liposome interior by simultaneous efflux of cations accompanying the influx of the drug, and possibly even formation of a self-perpetuating $\mathrm{pH}$ gradient to drive progressive drug accumulation. This may help explain the remarkably high loading capacity of triethylammonium sucrose octasulfate liposomes, with 800 $\mathrm{g}$ of irinotecan per mole of phospholipid (corresponding to 109,000 drug molecules per particle).

\section{Preclinical evaluation of nal-IRI}

Pharmacokinetic testing of nal-IRI in normal female rats demonstrated high in vivo stability of the liposomal construct, with significantly longer circulation times when compared to administration of free drug (half-life $\left[t_{1 / 2}\right]$ $=10.7$ hours compared to 0.27 hours, respectively). ${ }^{35}$ Almost one-quarter $(23.2 \%)$ of the injected dose of nal-IRI was detectable in circulation at 24 hours, compared to free CPT-11 (irinotecan), of which $98 \%$ is cleared within 30 minutes. Moreover, with the nal-IRI formulation there was less premature conversion to $\mathrm{SN}-38$, due in part to slow release of irinotecan from liposomes $\left(t_{1 / 2}\right.$ for irinotecan release $=56.8$ hours $)$. When tested in mice, nal-IRI showed dramatic regressions in a breast (BT474) tumor xenograft model with a $100 \%$ cure rate, without significant corresponding treatment-related toxicities aside from transient weight loss. Antitumor activity in a colon cancer (HT29) xenograft model was also observed, albeit not quite to as striking a degree.

Hann et $\mathrm{al}^{36}$ were the first to evaluate nal-IRI in pancreatic cancer, in the context of a bioluminescent-based orthotopic xenograft model (COLO357/L3.6pl). Compared to the equivalent dose of free drug, nal-IRI (as well as a separate, novel EGFR-targeted immunoliposomal form of irinotecan) showed superior antitumor activity, including a number of durable tumor regressions, without any significant systemic toxicity.
In a more recently published study, Kalra et $\mathrm{al}^{37}$ measured irinotecan and SN-38 levels in both plasma and tumor in mice harboring a variety of cell-line and patient-derived tumor xenografts (colorectal, ovarian, lung, and pancreatic) following administration of either nal-IRI or free irinotecan. Consistent with prior findings, plasma levels of both irinotecan and $\mathrm{SN}-38$ persisted much longer in circulation ( $>50$ hours) following administration of nal-IRI compared to free irinotecan. Similarly, prolonged exposure within tumors of both irinotecan and SN-38 was observed following nal-IRI administration; levels of both were still detectable at 168 hours, far longer than free irinotecan, where tumoral clearance of drug and active metabolite was noted by 24-48 hours. Ultimately, using model sensitivity analyses, these investigators determined that tumor SN-38 duration - reflecting both drug deposition and local activation of irinotecan by carboxylesterases to its active metabolite - represents the key driver of in vivo sensitivity to irinotecan-based treatment. Hence, the superior antitumor activity of nal-IRI could be attributed to its superior pharmacokinetic properties, with the ability of this liposomal construct to prolong drug exposure within tumors, compared to free irinotecan.

\section{Clinical development of nal-IRI}

The first-in-human clinical trial of nal-IRI, conducted in Taiwan by Chang et al, ${ }^{38}$ consisted of a Phase I dose-escalation study in patients with advanced refractory solid tumors, including cervical, breast, neuroendocrine, pancreatic, non-smallcell lung, and thymic cancers. In total, eleven patients were enrolled across three dose levels, with the maximal tolerated dose (MTD) established at $120 \mathrm{mg} / \mathrm{m}^{2}$ on an every-3-week schedule. DLTs at the next higher dose level $\left(180 \mathrm{mg} / \mathrm{m}^{2}\right)$ included grade 4 neutropenia in one patient and grade 4 febrile neutropenia, grade 4 thrombocytopenia with bleeding, and grade 4 diarrhea in a second patient resulting in death from septicemia and disseminated intravascular coagulation. Pharmacokinetic analyses demonstrated slow clearance, small volume of distribution, and prolonged terminal half-life of nal-IRI - all findings consistent with preclinical PK studies of nal-IRI in mice and rats - with a plasma concentrationtime profile approximately matching that of total irinotecan, reflecting the slow release of free-form irinotecan from the nanoliposomal carrier over time. The area under the curve of SN-38 at MTD of nal-IRI was comparable to historic data for conventional irinotecan dosed at $300-350 \mathrm{mg} / \mathrm{m}^{2}$. Of the six patients enrolled at MTD, gastrointestinal toxicities were frequently observed, including grade $3 / 4$ diarrhea in two patients 
$(33.3 \%)$ and grade $3 / 4$ vomiting in four patients $(66.7 \%)$. Two of ten $(20 \%)$ response-evaluable patients demonstrated a partial response by RECIST (Response Evaluation Criteria in Solid Tumors); notably, this included the one patient with pancreatic cancer treated at $180 \mathrm{mg} / \mathrm{m}^{2}$ who had failed several prior lines of chemotherapy.

A number of Phase I, II, and III clinical trials of nal-IRI have since been conducted across a variety of solid tumors, with a particular focus on those disease indications in which irinotecan has demonstrable clinical activity. A summary of these studies is listed in Table 1 . Chen et $\mathrm{al}^{39}$ were the first to report (in abstract form) the feasibility of combining nal-IRI with a second chemotherapeutic agent, 5-FU, in a dose-finding Phase I trial in patients with heavily pretreated solid tumors. The MTD using an every-3-week dosing schedule consisted of nal-IRI $80 \mathrm{mg} / \mathrm{m}^{2}$ (day 1) plus 24-hour infusional 5-FU 2,000 mg/m² and LV $200 \mathrm{mg} / \mathrm{m}^{2}$ (days 1 and 8). DLTs at higher doses of nal-IRI included diarrhea and cytopenias. The best response of 15 evaluable patients was partial response in two patients (gastric cancer and breast cancer) and stable disease in an additional nine.

Given the proven efficacy of irinotecan in colorectal cancer, there has naturally been considerable interest in studying nal-IRI for this indication to see if it compares favorably to the free-form drug. The first trial of nal-IRI specific for advanced colorectal cancer evaluated a biweekly schedule of the drug administered as monotherapy in patients who had failed first-line oxaliplatin-based therapy, with a dose of $100 \mathrm{mg} / \mathrm{m}^{2}$ established as the MTD. ${ }^{40}$ Four of 17 evaluable patients $(23.5 \%)$ achieved a partial response while an additional eight showed stable disease, for an overall disease control rate of $70.6 \%$. These promising results prompted a subsequent randomized Phase II study, the PEPCOL trial, in a similar patient population conducted by the Groupe Coopérateur Multidisciplinaire en Oncologie (GERCOR) in France. ${ }^{41}$ In this trial, 55 patients with advanced colorectal cancer were randomized in a 1:1 fashion to receive either nal-IRI plus 5-FU/LV administered at a biweekly dose schedule (referred to in this study as FUPEP) or 5-FU/LV plus irinotecan (FOLFIRI). While objective response rate was similar between the two arms (16.7\% for FUPEP, $11.5 \%$ for FOLFIRI), the toxicity profile favored FUPEP, with lower rates of grade $3-4$ diarrhea ( $21 \%$ vs $33 \%$ ) and neutropenia (11\% vs $30 \%$ ). Importantly, on the basis of these results, most notably the attractive safety profile, the decision was made to add this combination (herein referred to as 5-FU/ LV/nal-IRI) as a third arm to the NAPOLI-1 Phase III trial in metastatic pancreatic cancer, as will be discussed.

One other study of nal-IRI in gastric and gastroesophageal junction (GEJ) cancer ${ }^{42}$ warrants mention here as it represents the largest published clinical trial of this agent at the time of this writing. This Phase II trial enrolled patients with locally advanced or metastatic gastric/GEJ adenocarcinomas who had progressed on frontline therapy, a clinical context in

Table I Completed and ongoing clinical studies of nal-IRI

\begin{tabular}{|c|c|c|c|}
\hline Indication & Regimen & Phase & Source \\
\hline Advanced solid tumors & nal-IRI q3 weeks & I & Chang et $\mathrm{al}^{38}$ \\
\hline Advanced solid tumors & $\begin{array}{l}\text { nal-IRI (day I) plus infusional 5-FU/LV } \\
\text { (days I and 8) q3 weeks }\end{array}$ & I & Chen et $\mathrm{al}^{39}$ \\
\hline Recurrent high-grade glioma & nal-IRI q3 weeks & I & Clarke et $\mathrm{al}^{49}$ \\
\hline Pediatric solid tumors & $\begin{array}{l}\text { nal-IRI plus cyclophosphamide (dosing } \\
\text { schedule not specified) }\end{array}$ & I & NCT02013336 \\
\hline $\begin{array}{l}\text { Metastatic colorectal cancer, following first-line } \\
\text { oxaliplatin-based chemotherapy }\end{array}$ & nal-IRI q2 weeks & II & Chen et $\mathrm{al}^{40}$ \\
\hline $\begin{array}{l}\text { Metastatic pancreatic cancer, } \\
\text { gemcitabine-refractory }\end{array}$ & nal-IRI q3 weeks & II & Ko et $\mathrm{al}^{45}$ \\
\hline $\begin{array}{l}\text { Metastatic gastric cancer, following first-line } \\
\text { chemotherapy }\end{array}$ & $\begin{array}{l}\text { nal-IRI q3 weeks (comparator arms: } \\
\text { irinotecan, docetaxel) }\end{array}$ & $\begin{array}{l}\text { II (3-arm } \\
\text { randomization) }\end{array}$ & Roy et $\mathrm{al}^{42}$ \\
\hline $\begin{array}{l}\text { Metastatic colorectal cancer, following first-line } \\
\text { oxaliplatin-based chemotherapy }\end{array}$ & $\begin{array}{l}\text { nal-IRI plus 5-FU/LV q2 weeks } \\
\text { (comparator arm: FOLFIRI) }\end{array}$ & $\begin{array}{l}\text { II (2-arm } \\
\text { randomization) }\end{array}$ & $\begin{array}{l}\text { Chibaudel } \\
\text { et } \mathrm{al}^{4 !}\end{array}$ \\
\hline Metastatic pancreatic cancer, untreated & $\begin{array}{l}\text { nal-IRI plus oxaliplatin and infusional } \\
\text { 5-FU/LV q2 weeks (comparator arms: } \\
\text { FOLFOX, gemcitabine/nab-paclitaxel) }\end{array}$ & $\begin{array}{l}\text { II (3-arm } \\
\text { randomization) }\end{array}$ & NCT0255 $\left.199\right|^{\mathrm{a}}$ \\
\hline $\begin{array}{l}\text { Metastatic pancreatic cancer, } \\
\text { gemcitabine-refractory }\end{array}$ & $\begin{array}{l}\text { nal-IRI q3 weeks } \\
\text { nal-IRI plus 5-FU/LV q2 weeks } \\
\text { (comparator arm: 5-FU/LV) }\end{array}$ & III & $\begin{array}{l}\text { von Hoff et } \mathrm{al}^{46} \text {, } \\
\text { Chen et } \mathrm{al}^{47}\end{array}$ \\
\hline
\end{tabular}

Note: ${ }^{\mathrm{T} T r i a l}$ ongoing.

Abbreviations: q3, every 3 weeks; q2, every 2 weeks; nal-IRI, nanoliposomal irinotecan; 5-FU, 5-fluorouracil; LV, leucovorin; FOLFIRI, 5-FU/LV plus irinotecan; FOLFOX, 5-FU/LV plus oxaliplatin. 
Table 2 Comparison of pharmacokinetic properties of nal-IRI vs free irinotecan

\begin{tabular}{lll}
\hline Parameter & $\begin{array}{l}\text { Free } \\
\text { irinotecan }\end{array}$ & $\begin{array}{l}\text { Nanoliposomal } \\
\text { irinotecan }\end{array}$ \\
\hline$C_{\text {max }}(\mathrm{ng} / \mathrm{mL})$ & 4,265 & 60,842 \\
$T_{\text {max }}($ hours $)$ & 1.6 & 2.1 \\
$\mathrm{AUC}_{0-\mathrm{t}}(\mathrm{hr} \cdot \mathrm{ng} / \mathrm{mL})$ & 24,155 & $1,651,508$ \\
$\mathrm{AUC}_{0-\infty}(\mathrm{hr} \cdot \mathrm{ng} / \mathrm{mL})$ & 26,159 & $1,812,221$ \\
$\mathrm{CL}\left(\mathrm{mL} / \mathrm{h} / \mathrm{m}^{2}\right)$ & 12,886 & 191 \\
$t_{1 / 2}($ hours $)$ & 7.7 & 21.2 \\
\hline
\end{tabular}

Note: Adapted from Roy AC, Park SR, Cunningham D, et al. A randomized phase Il study of PEPO2 (MM-398), irinotecan or docetaxel as a second-line therapy in patients with locally advanced or metastatic gastric or gastro-oesophageal junction adenocarcinoma. Ann Oncol. 2013;24(6):1567-1573.42 By permission of Oxford University Press on behalf the European Society for Medical Oncology.

Abbreviations: nal-IRI, nanoliposomal irinotecan; $C_{\max }$, peak plasma concentration $T_{\max }$, time to reach $C_{\max } ; A \cup C$, area under the curve; $C L$, clearance; $t_{1 / 2}$, elimination half-life.

which single-agent irinotecan represents one of the standards of care. Patients were randomized in 1:1:1 fashion to receive nal-IRI, irinotecan, or docetaxel ( $\mathrm{n}=44$ in each arm). Objective response rate, which represented the primary study endpoint, was similar for nal-IRI (13.6\%) and docetaxel (15.9\%), both of which were higher than for irinotecan $(6.8 \%)$. Median progression-free and overall survival rates were similar across all three arms. This study was also informative in allowing the opportunity to directly compare the toxicity profile of nal-IRI (dosed at $120 \mathrm{mg} / \mathrm{m}^{2}$ in this trial) with that of standard irinotecan $\left(300 \mathrm{mg} / \mathrm{m}^{2}\right)$. Rates of grade $3-4$ adverse events were similar between these two treatment arms $(38.6 \%$ vs $34.1 \%)$, as was diarrhea of any grade $(72.7 \%$ vs $68.2 \%)$. Additionally, similar to prior preclinical and clinical studies, nal-IRI also demonstrated favorable pharmacokinetic properties, including a larger area under the curve, lower clearance, and smaller volume of distribution for total irinotecan when compared to free irinotecan. A summary comparing the major pharmacokinetic properties of nal-IRI and free irinotecan is provided in Table 2.

\section{nal-IRI in pancreatic cancer}

The track record of irinotecan in pancreatic cancer clinical trials has a somewhat checkered past. As noted earlier, irinotecan comprises a key component of the FOLFIRINOX regimen that now represents a gold standard in the frontline setting. Conversely, in two randomized Phase III trials for metastatic pancreatic cancer, the addition of irinotecan to gemcitabine did not confer any survival benefit. ${ }^{43,44}$ Other smaller Phase II trials evaluating irinotecan alone and in combination with other agents, in particular fluoropyrimidines, have demonstrated modest activity in pancreatic cancer, most commonly in the postgemcitabine setting (Table 3).

Ko et $\mathrm{a}^{45}$ conducted the first pancreas-specific trial of nal-IRI, consisting of a multinational Phase II study in patients with metastatic pancreatic cancer who had progressed on frontline gemcitabine-based chemotherapy. For this singlearm trial, conducted in the United States and Taiwan, forty patients received single-agent nal-IRI on an every 3-week schedule. Starting doses were $120 \mathrm{mg} / \mathrm{m}^{2}$, with the option of dose-escalating to $150 \mathrm{mg} / \mathrm{m}^{2}$ if the first cycle was welltolerated. Overall, 26 patients (65\%) experienced at least one treatment-emergent adverse event categorized as grade 3 or higher by National Cancer Institute Common Toxicity Criteria (NCI-CTC) criteria. Most frequent grade 3-5 toxicities included neutropenia (30\%), fatigue/asthenia (20\%), diarrhea $(15 \%)$, nausea $(10 \%)$, and anorexia $(10 \%)$. Also of particular note was three patients $(7.5 \%)$ who died related to complications of treatment, including aspiration pneumonia, sepsis, and respiratory failure, all developed in the setting of neutropenia. In terms of efficacy, three patients achieved a partial response as evaluated by RECIST criteria, with an additional $17(42.5 \%)$ demonstrating stable disease for a minimum of two cycles. Ten patients (31.3\%) experienced a CA19-9 response, defined as a decline in this tumor marker by $50 \%$ or greater for those with baseline levels greater than two times

Table 3 Select studies of irinotecan, alone or in combination with a fluoropyrimidine, in advanced pancreatic cancer

\begin{tabular}{llllll}
\hline Regimen & Sample size & RR (\%) & PFS/TTP (months) & Median survival (months) & Source \\
\hline Irinotecan & 33 & 9 & 2.0 & 6.6 & Yi et al $^{50}$ \\
Irinotecan $^{\text {FOLFIRI }}{ }^{2}$ & 56 & 3.6 & 2.9 & 5.3 & Takahara et al $^{51}$ \\
FOLFIRI & 40 & 37.5 & 5.6 & 12.1 & Taieb et al $^{52}$ \\
FOLFIRI & 31 & 0 & 1.9 & 3.9 & Yoo et al $^{53}$ \\
FOLFIRI or XELIRI & 40 & 15 & 3.7 & 6.0 & $\mathrm{Gebbia} \mathrm{et} \mathrm{al}^{54}$ \\
FOLFIRI & 34 & 0 & 2.0 & 4.2 & $\mathrm{Cereda} \mathrm{et} \mathrm{al}^{55}$ \\
FOLFIRI & 50 & 8.0 & 3.2 & 5.0 & Zaniboni et al $^{56}$ \\
Irinotecan plus S-I & 63 & 7.9 & 3.0 & 6.6 & Neuzillet et al $^{57}$ \\
\hline
\end{tabular}

Notes: aEvaluated in first-line setting; all other studies were conducted in previously treated patients.

Abbreviations: RR, relative risk; PFS, progression-free survival; TTP, time to progression; FOLFIRI, 5-FU/LV plus irinotecan; nal-IRI, nanoliposomal irinotecan; 5-FU, 5-fluorouracil; LV, leucovorin. 
the upper limits of normal. Median progression-free and overall survival for the entire cohort was 2.4 and 5.2 months, respectively, with a 6-month survival rate of $42.5 \%$.

Based on this signal of activity, a global Phase III, randomized, open-label trial called NAPOLI-1 (von Hoff et al; ${ }^{46}$ updated in Chen et $\mathrm{al}^{47}$ ) was designed to formally evaluate the efficacy of nal-IRI for patients with metastatic pancreatic cancer previously treated with a gemcitabine-based regimen (one or more prior lines of therapy allowed). The study was originally intended to provide a direct head-to-head comparison between two arms: nal-IRI at the same dose schedule used in the Phase II trial (120 mg/m² every 3 weeks), and a control arm of 5-FU (administered as a 24-hour infusion at $\left.2,000 \mathrm{mg} / \mathrm{m}^{2}\right)$ plus LV (200 mg/m²), administered weekly for 4 out of 6 weeks. However, shortly after the study began enrollment, results of the PEPCOL colorectal trial ${ }^{41}$ became available suggesting a favorable safety profile of biweekly nal-IRI plus infusional 5-FU and LV; on this basis, the trial design was modified to include a third arm consisting of the combination of nal-IRI $\left(80 \mathrm{mg} / \mathrm{m}^{2}\right), 46$-hour infusion of 5-fluorouracil $\left(2,400 \mathrm{mg} / \mathrm{m}^{2}\right)$, and LV (400 mg/m²), administered in 2-week cycles. In total, 417 patients were enrolled across the three treatment arms.

The main safety and efficacy findings of NAPOLI-1 are summarized in Table 4. Gastrointestinal-related toxicities, including nausea, vomiting, and diarrhea, were the most common adverse events reported, and occurred more frequently in the two nal-IRI-containing arms compared to the 5-FU/LV alone arm (with the highest incidence observed in the nal-IRI monotherapy arm). The majority of these were grades 1 and 2, although grade 3 or 4 diarrhea was reported in $21 \%$ of patients receiving nal-IRI alone and $13 \%$ in those receiving nal-IRI plus 5-FU/LV. Complications of neutropenia (neutropenic sepsis, febrile neutropenia) occurred in less than $5 \%$ of patients on either nal-IRI-containing arm, vs no reported cases on the 5-FU/LV alone arm. In total, five deaths attributed to study treatment were reported, four in the nal-IRI arm (gastrointestinal toxicity, infectious enterocolitis, septic shock, and disseminated intravascular coagulation with pulmonary embolism) and one in the nal-IRI plus 5-FU/LV arm (septic shock).

In the entire (intention-to-treat) cohort, patients on the nal-IRI plus 5-FU/LV arm demonstrated statistically significant improvements compared with those on the 5-FU/ LV alone arm in terms of all clinical relevant parameters, as shown in Table 3. Hazard ratios for median OS, PFS, and time to treatment failure were $0.67,0.56$, and 0.6 , respectively, all statistically significant. Significantly higher rates of objective radiographic response and CA $19-9$ decline $\geq 50 \%$ were also observed. Meanwhile, while nal-IRI alone also produced significantly higher rates of radiographic and biomarker response compared to $5-\mathrm{FU} / \mathrm{LV}$, overall and progressionfree survival rates were not significantly higher (HR for OS and PFS $=0.99$ and 0.81 , respectively). Forest plot analyses showed that survival benefit for the MM-398-containing combination was maintained across all subgroups, including those with lower Karnofsky performance status (KPS 70-80), age greater than 65 years old, and receiving two or more prior lines of systemic therapy.

On the basis of these positive results, the FDA in October 2015 approved nal-IRI in combination with 5-FU/ $\mathrm{LV}$ for the treatment of patients with metastatic pancreatic cancer following disease progression on gemcitabine-based therapy. This represented a landmark of sorts, as it became

Table 4 Safety and efficacy results of the Phase III NAPOLI-I trial

\begin{tabular}{|c|c|c|c|c|c|c|}
\hline \multirow[t]{2}{*}{ Adverse event (\%) } & \multicolumn{2}{|c|}{ nal-IRI +5-FU/LV (n=II 7) } & \multicolumn{2}{|c|}{ 5-FU/LV (n=I49) } & \multicolumn{2}{|c|}{ nal-IRI $(n=\mid 5 I)$} \\
\hline & Any grade & Grade 3/4 & Any grade & Grade 3/4 & Any grade & Grade $3 / 4$ \\
\hline \multicolumn{7}{|l|}{ Safety } \\
\hline Diarrhea & 59 & 13 & 26 & 5 & 70 & 21 \\
\hline Vomiting & 52 & II & 26 & 3 & 54 & 14 \\
\hline Nausea & 51 & 8 & 34 & 3 & 61 & 5 \\
\hline Decreased appetite & 44 & 4 & 32 & 2 & 49 & 19 \\
\hline Fatigue & 40 & 14 & 28 & 4 & 37 & 6 \\
\hline Neutropenia & 39 & 27 & 5 & 2 & 25 & 15 \\
\hline \multicolumn{7}{|l|}{ Efficacy } \\
\hline Median overall survival (months) & 6.1 & & 4.2 & & 4.9 & \\
\hline Median progression-free survival (months) & 3.1 & & 1.5 & & 2.7 & \\
\hline Median time to treatment failure (months) & 2.3 & & 1.4 & & 1.7 & \\
\hline Objective response rate $(\%)$ & 16 & & I & & 6 & \\
\hline CA $19-9$ reduction $\geq 50 \%(\%)$ & 29 & & 10 & & 24 & \\
\hline
\end{tabular}

Notes: Data from von Hoff D, Dhindsa N, Bayever E, et al. ${ }^{46}$, Chen L, Von Hoff D, Li C, et al. ${ }^{47}$

Abbreviations: nal-IRI, nanoliposomal irinotecan; 5-FU, 5-fluorouracil; LV, leucovorin; CAI9-9, carbohydrate antigen I9-9. 
the first therapeutic agent to receive approval in this disease specifically for use in the second-line setting and beyond.

\section{Future directions}

The approval of nal-IRI raises a number of practical issues as well as considerations that may inform future clinical trial design. First of all, selection of the individuals most likely to benefit from, and tolerate, nal-IRI requires further clarification and refinement. The FDA-approved indication specifies this drug should be limited to those patients who have received prior gemcitabine-based treatment; at present, this would most commonly consist of the combination of gemcitabine and nab-paclitaxel, or less commonly another gemcitabine-based doublet or monotherapy. On the other hand, whether nal-IRI plus 5-FU/LV has any activity following first-line treatment with FOLFIRINOX, the other first-line standard for metastatic pancreatic cancer, is unknown; to date, we have no knowledge regarding whether the superior biodistribution and pharmacokinetic properties of nal-IRI can overcome resistance to a standard irinotecancontaining regimen. This highlights a related question: how does nal-IRI compare to standard irinotecan in pancreatic cancer? It seems unlikely that a randomized clinical trial directly comparing FOLFIRI to nal-IRI plus 5-FU/LV will ever be performed, and so we are left using historic FOLFIRI data from relatively small patient cohorts to make (admittedly inexact) cross-study comparisons regarding their respective efficacies in this disease setting.

Furthermore, does the nal-IRI-based combination now supplant the prior de facto second-line standard of care, FOLFOX, for the treatment of metastatic pancreatic cancer? Again, a head-to-head comparison of these two regimens has not been formally performed. One might conceivably develop a biomarker- or pharmacogenetically-driven approach to guide selection of therapy (eg, to choose between an irinotecan- vs platinum-based regimen). It is well-known, for instance, that genetic variants in isoforms of the enzyme UDP-glucuronosyltransferase, in particular UGT1A1, contribute to variability in the metabolism and excretion of irinotecan, producing significant interpatient differences in drug-related toxicity. The most common example of this is UGT1A1*28; individuals who are homozygous for this allele (also known as 7/7, based on the number of repeats of the two-base insertion TA in the promoter region of the gene), which is associated with severely reduced enzymatic activity, are more than threefold likely to develop severe neutropenia following irinotecan-based therapy compared to those with wild genotype (reviewed in Palomaki et $\mathrm{al}^{48}$ ). While relatively scant pharmacogenetic data are available from prior nal-IRI studies to provide guidance, it would be reasonable to use UGT1A1 genotyping to help in this decision-making process. For example, those individuals harboring genetic polymorphisms known to confer greater toxicity might proceed with nal-IRI at reduced starting doses, or even steer away from this agent, if they are particularly fragile and the risk of major toxicity is too high.

Certainly the other area ripe for consideration in future studies centers on the feasibility of combining nal-IRI with other drugs; this could entail either combinations with other cytotoxic agents, or using nal-IRI ( $\pm 5-\mathrm{FU} / \mathrm{LV})$ as a chemotherapy backbone upon which to add molecularly targeted agents or immunotherapies. Of particular interest is evaluation of nal-IRI in the frontline setting, most notably as part of an FOLFIRINOX regimen where nal-IRI is substituted for standard irinotecan. An ongoing clinical trial that recently opened in September 2015 is looking at this particular combination in newly diagnosed patients with metastatic disease (NCT02551991). Following a safety run-in to confirm the proper dosing and tolerability of nal-IRI in combination with oxaliplatin and 5-FU/LV, the study will then randomize patients to one of three arms: this triple-drug combination regimen, nal-IRI plus 5-FU/LV, or the combination of gemcitabine plus nab-paclitaxel.

\section{Conclusion}

In summary, nal-IRI is an important advance in the treatment landscape for metastatic pancreatic cancer. Not only does it represent the first drug specifically approved for use in the second-line setting and beyond in this disease, but nal-IRI also serves as an important proof of principle as the first liposomal formulation of an anticancer agent to gain an indication for any gastrointestinal malignancy. Taking advantage of its ability to deliver an increased drug payload to sites of disease, without concomitant increased systemic toxicity, nal-IRI offers several pharmacologic advantages over free irinotecan. Whether this success ushers in a new era of exploration of liposomal anticancer drugs, or other efficient delivery vehicles, remains to be seen. Successor studies will be critical not only to further define the appropriate use of nal-IRI in pancreatic and other camptothecin-sensitive malignancies, but also to explore predictive biomarkers of drug sensitivity as well as the pharmacodynamics effects of nal-IRI on the tumor and its microenvironment (which could be readily evaluated, for example, in the neoadjuvant setting). For now, from a practical perspective, nal-IRI represents a valuable step in allowing more patients with 
metastatic pancreatic cancer to be sequenced through two, or even more, lines of systemic therapy with the promise of clinically meaningful improvements in longevity.

\section{Disclosure}

The author has previously participated in several advisory board meetings for Merrimack. PharmaEngine provided financial support to his institution for conduct of the Phase II trial of nal-IRI in pancreatic cancer. The author reports no other conflicts of interest in this work.

\section{References}

1. Siegel RL, Miller KD, Jemal A. Cancer statistics, 2015. CA Cancer JClin. 2015;65(1):5-29.

2. Rahib L, Smith BD, Aizenberg R, Rosenzweig AB, Fleshman JM, Matrisian LM. Projecting cancer incidence and deaths to 2030: the unexpected burden of thyroid, liver, and pancreas cancers in the United States. Cancer Res. 2014;74(11):2913-2921.

3. Torre LA, Bray F, Siegel RL, Ferlay J, Lortet-Tieulent J, Jemal A. Global cancer statistics, 2012. CA Cancer J Clin. 2015;65(2):87-108.

4. Ko AH. Progress in the treatment of metastatic pancreatic cancer and the search for next opportunities. J Clin Oncol. 2015;33(16):1779-1786.

5. Burris HA III, Moore MJ, Andersen J, et al. Improvements in survival and clinical benefit with gemcitabine as first-line therapy for patients with advanced pancreas cancer: a randomized trial. J Clin Oncol. 1997; 15(6):2403-2413.

6. Moore MJ, Goldstein D, Hamm J, et al. Erlotinib plus gemcitabine compared with gemcitabine alone in patients with advanced pancreatic cancer: a phase III trial of the National Cancer Institute of Canada Clinical Trials Group. J Clin Oncol. 2007;25(15):1960-1966.

7. Conroy T, Desseigne F, Ychou M, et al. FOLFIRINOX versus gemcitabine for metastatic pancreatic cancer. N Engl J Med. 2011;364(19): 1817-1825.

8. Gourgou-Bourgade S, Bascoul-Mollevi C, Desseigne F, et al. Impact of FOLFIRINOX compared with gemcitabine on quality of life in patients with metastatic pancreatic cancer: results from the PRODIGE 4/ACCORD 11 randomized trial. J Clin Oncol. 2013;31(1):23-29.

9. Von Hoff D, Penny R, Shack S, et al. Frequency of potential therapeutic targets identified by immunohistochemistry (IHC) and DNA microarray (DMA) in tumors from patients who have progressed on multiple therapeutic agents [abstract 3071]. J Clin Oncol. 2006;24(Suppl 18):138s.

10. Von Hoff DD, Ramanathan RK, Borad MJ, et al. Gemcitabine plus nab-paclitaxel is an active regimen in patients with advanced pancreatic cancer: a phase I/II trial. J Clin Oncol. 2011;29(34):4548-4554.

11. Von Hoff DD, Ervin T, Arena FP, et al. Increased survival in pancreatic cancer with nab-paclitaxel plus gemcitabine. $N$ Engl J Med. 2013;369(18):1691-1703.

12. Assaf E, Verlinde-Carvalho M, Delbaldo C, et al. 5-fluorouracil/ leucovorin combined with irinotecan and oxaliplatin (FOLFIRINOX) as second-line chemotherapy in patients with metastatic pancreatic adenocarcinoma. Oncology. 2011;80(5-6):301-306.

13. Berger AK, Weber TF, Jager D, Springfeld C. Successful treatment with nab-paclitaxel and gemcitabine after FOLFIRINOX failure in a patient with metastasized pancreatic adenocarcinoma. Onkologie. 2013;36(12):763-765.

14. Bertocchi P, Abeni C, Meriggi F, et al. Gemcitabine plus nab-paclitaxel as second-line and beyond treatment for metastatic pancreatic cancer: a single institution retrospective analysis. Rev Recent Clin Trials. 2015; 10(2):142-145.

15. Lee MG, Lee SH, Lee SJ, et al. 5-fluorouracil/leucovorin combined with irinotecan and oxaliplatin (FOLFIRINOX) as second-line chemotherapy in patients with advanced pancreatic cancer who have progressed on gemcitabine-based therapy. Chemotherapy. 2013;59(4):273-279.
16. Portal A, Pernot S, Siauve N, et al. Sustained response with gemcitabine plus Nab-paclitaxel after folfirinox failure in metastatic pancreatic cancer: report of an effective new strategy. Clin Res Hepatol Gastroenterol. 2014;38(2):e23-e26.

17. Rahma OE, Duffy A, Liewehr DJ, Steinberg SM, Greten TF. Second-line treatment in advanced pancreatic cancer: a comprehensive analysis of published clinical trials. Ann Oncol. 2013;24(8):1972-1979.

18. Oettle H, Riess H, Stieler JM, et al. Second-line oxaliplatin, folinic acid, and fluorouracil versus folinic acid and fluorouracil alone for gemcitabine-refractory pancreatic cancer: outcomes from the CONKO003 trial. J Clin Oncol. 2014;32(23):2423-2429.

19. Gill S, Ko Y, Cripps M, et al. PANCREOX: a randomized phase 3 study of 5FU/LV with or without oxaliplatin for second-line advanced pancreatic cancer in patients who have received gemcitabine-based chemotherapy [abstract 4002]. J Clin Oncol. 2012;32(Suppl 15):5s.

20. Hurwitz HI, Uppal N, Wagner SA, et al. Randomized, double-blind, phase II study of ruxolitinib or placebo in combination with capecitabine in patients with metastatic pancreatic cancer for whom therapy with gemcitabine has failed. J Clin Oncol. 2015;33(34):4039-4047.

21. Clark CE, Beatty GL, Vonderheide RH. Immunosurveillance of pancreatic adenocarcinoma: insights from genetically engineered mouse models of cancer. Cancer Lett. 2009;279(1):1-7.

22. Le DT, Wang-Gillam A, Picozzi V, et al. Safety and survival with GVAX pancreas prime and Listeria Monocytogenes-expressing mesothelin (CRS-207) boost vaccines for metastatic pancreatic cancer. J Clin Oncol. 2015;33(12):1325-1333.

23. Tsai CS, Park JW, Chen LT. Nanovector-based therapies in advanced pancreatic cancer. J Gastrointest Oncol. 2011;2(3):185-194.

24. Bertrand N, Wu J, Xu X, Kamaly N, Farokhzad OC. Cancer nanotechnology: the impact of passive and active targeting in the era of modern cancer biology. Adv Drug Deliv Rev. 2014;66:2-25.

25. Hyodo K, Yamamoto E, Suzuki T, Kikuchi H, Asano M, Ishihara H. Development of liposomal anticancer drugs. Biol Pharm Bull. 2013; 36(5):703-707.

26. Matsumura $Y$, Maeda $H$. A new concept for macromolecular therapeutics in cancer chemotherapy: mechanism of tumoritropic accumulation of proteins and the antitumor agent smancs. Cancer Res. 1986;46(12 Pt 1): 6387-6392.

27. Senior JH. Fate and behavior of liposomes in vivo: a review of controlling factors. Crit Rev Ther Drug Carrier Syst. 1987;3(2):123-193.

28. Stathopoulos GP, Boulikas T, Vougiouka M, Rigatos SK, Stathopoulos JG. Liposomal cisplatin combined with gemcitabine in pretreated advanced pancreatic cancer patients: a phase I-II study. Oncol Rep. 2006;15(5): 1201-1204.

29. Loehr M, Bodoky G, Fölsch U, et al. Cationic liposomal paclitaxel in combination with gemcitabine in patients with advanced pancreatic cancer: a phase II trial [abstract 4526]. J Clin Oncol. 2009;27(Suppl):15s.

30. Pommier Y. Topoisomerase I inhibitors: camptothecins and beyond. Nat Rev Cancer. 2006;6(10):789-802.

31. Kawato Y, Aonuma M, Hirota Y, Kuga H, Sato K. Intracellular roles of SN-38, a metabolite of the camptothecin derivative CPT-11, in the antitumor effect of CPT-11. Cancer Res. 1991;51(16):4187-4191.

32. Colbern GT, Dykes DJ, Engbers C, et al. Encapsulation of the topoisomerase I inhibitor GL147211C in pegylated (STEALTH) liposomes: pharmacokinetics and antitumor activity in HT29 colon tumor xenografts. Clin Cancer Res. 1998;4(12):3077-3082.

33. Emerson DL, Bendele R, Brown E, et al. Antitumor efficacy, pharmacokinetics, and biodistribution of NX 211: a low-clearance liposomal formulation of lurtotecan. Clin Cancer Res. 2000;6(7):2903-2912.

34. Messerer CL, Ramsay EC, Waterhouse D, et al. Liposomal irinotecan: formulation development and therapeutic assessment in murine xenograft models of colorectal cancer. Clin Cancer Res. 2004;10(19): 6638-6649.

35. Drummond DC, Noble CO, Guo Z, Hong K, Park JW, Kirpotin DB. Development of a highly active nanoliposomal irinotecan using a novel intraliposomal stabilization strategy. Cancer Res. 2006;66(6): 3271-3277. 
36. Hann B, Peth K, Wang D, et al. Lipidic nanoparticle CPT-11 in a bioluminescent orthotopic pancreas cancer model [abstract 5648]. Paper presented at: AACR Annual Meeting, April 14-18, 2007, Los Angeles, CA.

37. Kalra AV, Kim J, Klinz SG, et al. Preclinical activity of nanoliposomal irinotecan is governed by tumor deposition and intratumor prodrug conversion. Cancer Res. 2014;74(23):7003-7013.

38. Chang TC, Shiah HS, Yang CH, et al. Phase I study of nanoliposomal irinotecan (PEP02) in advanced solid tumor patients. Cancer Chemother Pharmacol. 2015;75(3):579-586.

39. Chen L, Shiah H, Chao T, et al. Phase I study of liposome irinotecan (PEP02) in combination with weekly infusion of 5-FU/LV in advanced solid tumors [abstract e13024]. J Clin Oncol. 2010;28(Suppl 15).

40. Chen L, Shiah H, Lin P, et al. Phase I study of biweekly liposome irinotecan (PEP02, MM-398) in metastatic colorectal cancer failed on first-line oxaliplatin-based chemotherapy [abstract 613]. J Clin Oncol. 2012;30(Supp1 4).

41. Chibaudel B, Maindrault-Goebel F, André T, et al. PEPCOL: a randomized noncomparative phase II study of PEP02 (MM-398) or irinotecan in combination with leucovorin and 5-fluorouracil as second-line therapy in patients with unresectable metastatic colorectal cancer - a GERCOR Study [abstract 751]. J Clinical Oncol. 2015;33(Suppl 3).

42. Roy AC, Park SR, Cunningham D, et al. A randomized phase II study of PEP02 (MM-398), irinotecan or docetaxel as a second-line therapy in patients with locally advanced or metastatic gastric or gastro-oesophageal junction adenocarcinoma. Ann Oncol. 2013;24(6):1567-1573.

43. Rocha Lima CM, Green MR, Rotche R, et al. Irinotecan plus gemcitabine results in no survival advantage compared with gemcitabine monotherapy in patients with locally advanced or metastatic pancreatic cancer despite increased tumor response rate. J Clin Oncol. 2004; 22(18):3776-3783

44. Stathopoulos GP, Syrigos K, Aravantinos G, et al. A multicenter phase III trial comparing irinotecan-gemcitabine (IG) with gemcitabine $(\mathrm{G})$ monotherapy as first-line treatment in patients with locally advanced or metastatic pancreatic cancer. Br J Cancer. 2006;95(5):587-592.

45. Ko AH, Tempero MA, Shan YS, et al. A multinational phase 2 study of nanoliposomal irinotecan sucrosofate (PEP02, MM-398) for patients with gemcitabine-refractory metastatic pancreatic cancer. Br J Cancer. 2013;109(4):920-925.

46. von Hoff D, Dhindsa N, Bayever E, et al. NAPOLI-1: randomized phase 3 study of MM-398 (nal-IRI), with or without 5-fluorouracil and leucovorin, versus 5-fluorouracil and leucovorin, in metastatic pancreatic cancer progressed on or following gemcitabine-based therapy [abstract]. Ann Oncol. 2014;25(Suppl 2):ii105-ii117.

47. Chen L, Von Hoff D, Li C, et al. Expanded analyses of napoli-1: phase 3 study of MM-398 (nal-IRI), with or without 5-fluorouracil and leucovorin, versus 5-fluorouracil and leucovorin, in metastatic pancreatic cancer previously treated with gemcitabine-based therapy [abstract 234]. J Clin Oncol. 2015;33(Suppl 3).
48. Palomaki GE, Bradley LA, Douglas MP, Kolor K, Dotson WD. Can UGT1A1 genotyping reduce morbidity and mortality in patients with metastatic colorectal cancer treated with irinotecan? An evidence-based review. Genet Med. 2009;11(1):21-34.

49. Clarke J, Molinaro A, DeSilva A, et al. A phase I trial of intravenous liposomal irinotecan in patients with recurrent high-grade gliomas [abstract 2029]. J Clin Oncol. 2015;33(Suppl 15).

50. Yi SY, Park YS, Kim HS, et al. Irinotecan monotherapy as second-line treatment in advanced pancreatic cancer. Cancer Chemother Pharmacol. 2009;63(6):1141-1145.

51. Takahara N, Nakai Y, Isayama H, et al. Uridine diphosphate glucuronosyl transferase 1 family polypeptide A1 gene (UGT1A1) polymorphisms are associated with toxicity and efficacy in irinotecan monotherapy for refractory pancreatic cancer. Cancer Chemother Pharmacol. 2013; 71(1):85-92.

52. Taieb J, Lecomte T, Aparicio T, et al. FOLFIRI.3, a new regimen combining 5-fluorouracil, folinic acid and irinotecan, for advanced pancreatic cancer: results of an Association des Gastro-Enterologues Oncologues (Gastroenterologist Oncologist Association) multicenter phase II study. Ann Oncol. 2007;18(3):498-503.

53. Yoo C, Hwang JY, Kim JE, et al. A randomised phase II study of modified FOLFIRI.3 vs modified FOLFOX as second-line therapy in patients with gemcitabine-refractory advanced pancreatic cancer. Br J Cancer. 2009;101(10):1658-1663.

54. Gebbia V, Maiello E, Giuliani F, Borsellino N, Arcara C, Colucci G. Irinotecan plus bolus/infusional 5-Fluorouracil and leucovorin in patients with pretreated advanced pancreatic carcinoma: a multicenter experience of the Gruppo Oncologico Italia Meridionale. Am J Clin Oncol. 2010; 33(5):461-464.

55. Cereda S, Reni M, Rognone A, et al. XELIRI or FOLFIRI as salvage therapy in advanced pancreatic cancer. Anticancer Res. 2010;30(11): 4785-4790.

56. Zaniboni A, Aitini E, Barni S, et al. FOLFIRI as second-line chemotherapy for advanced pancreatic cancer: a GISCAD multicenter phase II study. Cancer Chemother Pharmacol. 2012;69(6):1641-1645.

57. Neuzillet C, Hentic O, Rousseau B, et al. FOLFIRI regimen in metastatic pancreatic adenocarcinoma resistant to gemcitabine and platinumsalts. World J Gastroenterol. 2012;18(33):4533-4541.

58. Mizuno N, Yamao K, Komatsu Y, et al. Randomized phase II trial of S-1 versus S-1 plus irinotecan (IRIS) in patients with gemcitabine-refractory pancreatic cancer [abstract 263]. J Clin Oncol. 2013;30(Suppl 4)
International Journal of Nanomedicine

\section{Publish your work in this journal}

The International Journal of Nanomedicine is an international, peerreviewed journal focusing on the application of nanotechnology in diagnostics, therapeutics, and drug delivery systems throughout the biomedical field. This journal is indexed on PubMed Central, MedLine, CAS, SciSearch $®$, Current Contents $\AA /$ Clinical Medicine,

\section{Dovepress}

Journal Citation Reports/Science Edition, EMBase, Scopus and the Elsevier Bibliographic databases. The manuscript management system is completely online and includes a very quick and fair peer-review system, which is all easy to use. Visit http://www.dovepress.com/ testimonials.php to read real quotes from published authors. 